\title{
Serological diagnosis of Trypanosoma cruzi: evaluation of three enzyme immunoassays and an indirect immunofluorescent assay
}

\section{Correspondence \\ Christine M. Litwin \\ christine.litwin@path.utah.edu}

Received 6 May 2005

Accepted 30 September 2005

\author{
Annette K. Malan, ${ }^{1}$ Erick Avelar, ${ }^{2}$ Sheldon E. Litwin, ${ }^{2}$ Harry R. Hill ${ }^{1,3}$ \\ and Christine M. Litwin ${ }^{1,3}$
}
${ }^{1}$ University of Utah, Associated Regional and University Pathologists (ARUP) Institute for Clinical and Experimental Pathology, Salt Lake City, Utah, USA
${ }^{2,3}$ Department of Medicine, Division of Cardiology ${ }^{2}$ and Departments of Pathology, Pediatrics and Medicine ${ }^{3}$, University of Utah School of Medicine, Salt Lake City, Utah, USA

\begin{abstract}
Chagas' disease is an important cause of heart failure in Latin America, but is rare in the United States. The immigration of persons from endemic countries increases the potential of encountering patients with the disease. Concerns have also been raised about the introduction of Trypanosoma cruzi, the parasite that causes the disease, into the blood supply and during organ transplantation. To compare Chagas' antibody tests that are available in the United States, we evaluated three IgG ELISAs, CeLLabs T. cruzi ELISA, Hemagen Chagas' kit and IVD Research Chagas' Serum Microwell ELISA, and MarDx indirect immunofluorescent assays. The CeLLabs and Hemagen IgG ELISAs had $100 \%$ agreement, sensitivity and specificity. The IVD Research IgG ELISA had $94.6 \%$ agreement, $100 \%$ sensitivity and $93 \%$ specificity.
\end{abstract}

\section{INTRODUCTION}

Chagas' disease is caused by the protozoan parasite Trypanosoma cruzi. The parasite is introduced into the host via triatomid insect vectors. Infected reduviid bugs spread the infection to man by depositing faeces on the skin; the faeces is then accidentally rubbed into the bite wound produced by the infected insect, an open cut or mucosal surfaces. Upon initial infection, humans generally experience mild symptoms such as fever or malaise (Kirchhoff, 1996). At the bite site, an erythematous and indurated swelling called a chagoma may appear, along with regional lymphadenopathy. If the entry site is through the conjunctiva, unilateral painless periorbital oedema known as Romaña's sign may occur (Krauss et al., 2003). T. cruzi is only fatal in $10 \%$ of acute infections (Schechter et al., 1983), usually after causing meningoencephalitis or congestive heart failure (Krauss et al., 2003; Tanowitz et al., 1992).

After 2-3 months, the infection enters an indeterminate stage that can last for decades. Eventually, $10-30 \%$ of infected individuals develop a chronic infection (WHO, 1991). Cardiomyopathy, megacolon and megaesophagus (Oelemann et al., 1998) are frequent complications, and

Abbreviations: ECG, electrocardiogram; EIA, enzyme immunoassay; FDA, Food and Drug Administration; IFA, immunofluorescent assay; SLE, systemic lupus erythematosus. lead to the demise of approximately 50000 people per year (WHO, 1991).

Although vector to human transmission is the best understood, oral (Shikanai-Yasuda et al., 1991) and congenital transmission (Freilij \& Altcheh, 1995), and infections via blood transfusion (Schmunis, 1991) and organ transplantation (CDC, 2002) have also been described. Transfusionrelated infection is also a concern in the United States, where an estimated 50000-100000 Latin American immigrants are infected with T. cruzi (Kirchhoff, 1993). Transfusion infections in the United States are well documented (Kirchhoff, 1996). Seroepidemiology studies have shown that $1 \cdot 1 \%$ of blood donors in Los Angeles, California had antibodies against T. cruzi (Kerndet et al., 1991), while Leiby et al. (1997) estimated that $2.5 \%$ of blood donors nationwide could have potentially been exposed to T. cruzi. Out of concern over the risk of future transfusion-related infections, the American Red Cross is preparing to screen all donated blood for T. cruzi, pending a Food and Drug Administration (FDA) approval of a test specific for screening blood donors. It is predicted that such a test will be approved in 2-3 years (McCarthy, 2003).

In order to assist physicians, who may treat either blood donors with positive test results or patients from T. cruzi endemic areas with chronic Chagas' disease, reference laboratories should prepare a testing algorithm for the diagnosis of Chagas' disease. Numerous studies addressing 
the performance of antibody assays have been published, most of which were carried out in Latin America (Avila et al., 1993; Ferreira et al., 2001; Leiby et al., 2000; Mendes et al., 1997; Oelemann et al., 1998; Reiche et al., 1998). Unfortunately, the assays that were previously evaluated either are no longer being produced by the respective manufacturer or are not available in the United States. In the present study, we evaluated the performance characteristics of three IgG ELISAs, and IgG and IgM indirect immunofluorescent assays (IFAs) that are available in the United States. We studied true T. cruzi antibody positive sera from patients at a Brazilian Chagas' disease clinic and control sera collected at our reference laboratory in Salt Lake City, Utah.

\section{METHODS}

\section{Human sera}

This study was approved by the Institutional Review Board of the University of Utah, IRB 12229. A total of 220 serum samples were used. The sera were subdivided into four groups

Group I. This group contained 30 samples from patients in Recife, Brazil with clinical evidence of $T$. cruzi infection [i.e. heart failure, syncope or arrhythmias, characteristic electrocardiogram (ECG) abnormalities, residence in a region endemic for T. cruzi or having a family member known to be infected; Table 1]. Frozen specimens from Oswaldo Cruz University hospital in Recife, Brazil, were shipped frozen and stored at $-20^{\circ} \mathrm{C}$ until ELISA or IFA testing commenced. Specimens were then stored at $4{ }^{\circ} \mathrm{C}$ while the evaluations were performed. Sixteen of the patients were asymptomatic, but resided in areas where Chagas' disease is endemic. Nine patients showed evidence of congestive heart failure. Three patients experienced palpitations and two patients were referred for further evaluation due to syncope (Table 1).

Group II. This group contained 37 samples from patients throughout the United States with serological evidence of parasitic infection by Cysticercosis $(n=7)$, Strongyloides $(n=3)$, Toxocara $(n=2)$, Entamoeba histolytica $(n=3)$, Leptospira $(n=1)$, Trichinella $(n=1)$, Malaria $(n=3)$, Schistosoma $(n=5)$, Leishmania $(n=2)$ or Toxoplasma gondii $(n=10)$.

Group III. This group contained 53 samples from patients throughout the United States with serological evidence of brucellosis, syphilis, infectious mononucleosis, rheumatoid arthritis, systemic lupus erythematosus (SLE) and rheumatic fever. Ten samples from each disease category were included in the group, except for brucellosis $(n=3)$.

Group IV. This group contained 100 samples collected from random healthy donors in the Salt Lake City, Utah area in 2002.

IgG and IgM IFA. All samples were tested for IgG and IgM antibodies against $T$. cruzi using an IFA slide supplied by MarDx Diagnostics. The antigen preparation consisted of epimastigotes of T. cruzi (Corpus Cristi strain), which had been cultured in a modification of a medium originally developed for the culture of Leishmania sp. A standardized amount of washed parasites were placed into the wells of specially prepared microscope slides. In the IgG assay, samples were diluted $1: 16$ in PBS diluent $[0.5 \%$ fetal bovine serum, $0 \cdot 1 \%$ thimerosal (Sigma) in PBS (Bio-Rad)] and added to IFA slides coated with a fixed antigen preparation of $T$. cruzi epimastigotes.
The slides were incubated in a moist chamber at room temperature for $30 \mathrm{~min}$ and then washed for $5 \mathrm{~min}$ in PBS. Fluorescein-labelled IgG conjugate, supplied by MarDx, was added to each reaction well and incubated at room temperature for $30 \mathrm{~min}$. After this second incubation, the slides were washed in PBS, coverslips were added and the slides examined at $\times 400$ magnification (numeric aperture $0.85 \mathrm{~mm}$ ) by fluorescent microscopy. Results were determined based on the fluorescence of the organisms in each sample well. Fluorescence was graded as recommended by the manufacturer, where a sample was reported positive if a $2+$ homogeneous surface fluorescence was observed in $>80 \%$ of the parasites in the well. Positive samples were serially diluted and titrated to end point. The reference interval for a positive result was $\geqslant 1: 16$.

Samples were assayed for IgM antibodies against T. cruzi in the same manner as described above, except that the initial dilution was performed in goat anti-human IgG diluent (Martins et al., 1995) and fluorescein-labelled IgM conjugate supplied by MarDx was added to each reaction well in the conjugation step. Samples were assayed three times on three consecutive days.

Commercial ELISA test systems. Three commercial ELISAs were evaluated in this study: the CeLLabs T. cruzi IgG ELISA (distributed by PANBIO), the FDA-cleared Hemagen Chagas' kit [enzyme immunoassay (EIA) method] (Columbia) and the IVD Research Chagas (T. cruzi) serum microwell ELISA (distributed by Biotech Trading Partners). The Hemagen EIA uses antigens from cultured T. cruzi epimastigotes. The IVD Research and CeLLabs EIAs use unspecified T. cruzi antigens. Testing was performed according to the manufacturers' instructions. Test results were analysed as recommended in the corresponding technical information for each assay. Samples that were discrepant between the assays were repeated in duplicate and reported as the mean $A_{450}$ value. Two positive samples and one negative sample were assayed three times in three consecutive days to evaluate reproducibility.

Statistical analysis. The clinical sensitivity of each assay was determined as the percentage of positive results with each test kit for the 30 known positive samples from Brazil. The clinical specificity of each assay was determined as the percentage of negative results with each test kit for the group IV samples (random healthy donors representing true negatives, $n=100$ ). The cross-reactivity of each assay was examined by testing sera from patients with other parasitic, bacterial and viral infections as well as a variety of autoimmune diseases (groups II and III). The $95 \%$ confidence intervals for the clinical sensitivity and specificity were also determined for each assay.

Reproducibility of $\boldsymbol{T}$. cruzi assays. The reproducibility of each assay was examined using serologically positive and negative sera repeatedly tested over 3 days. For the IgG IFA, one negative and two positive samples were assayed three times in duplicate. For the IgM IFA, one positive and two negative samples were assayed three times in duplicate. For the commercial ELISA test systems, two positive samples were assayed three times in replicates of five on three consecutive days. Where possible, inter-assay and intra-assay precision was determined.

\section{RESULTS AND DISCUSSION}

\section{Clinical information}

Clinical information for each patient in group I, along with their serological results, are shown in Table 1. The ages of the patients ranged from 25 to 71 (mean $49 \cdot 1$ ) years. Fiftythree per cent of the patients (16) were female. 
Table 1. Clinical symptoms and serological results for samples in group I

CeLLabs ELISA $A_{450}$ values for the different reference intervals are as follows: negative, $<0 \cdot 2$; equivocal, $0 \cdot 2-0 \cdot 3$; positive, $>0 \cdot 3$. Hemagen ELISA index values for the different reference intervals are as follows: negative, $<0 \cdot 276$; equivocal, $0 \cdot 277-0 \cdot 303$; positive, $\geqslant 0 \cdot 304$. IVD Research ELISA $A_{450}$ values for the different reference intervals are as follows: negative, $<0 \cdot 2$; positive, $\geqslant 0 \cdot 2$. IgG and IgM IFA reciprocal of titre values for the different reference intervals are as follows: negative, $<16$; positive, $\geqslant 16$.

\begin{tabular}{|c|c|c|c|c|c|c|c|c|c|}
\hline $\begin{array}{l}\text { Panel } \\
\text { no. }\end{array}$ & Age & Gender & $\begin{array}{c}\text { ECG } \\
\text { finding }\end{array}$ & $\begin{array}{l}\text { Main reason } \\
\text { for evaluation }\end{array}$ & \multicolumn{5}{|c|}{ Serology result } \\
\hline 171 & 25 & $\mathrm{~F}$ & Normal & Endemic area & $1 \cdot 67$ & $0 \cdot 96$ & $2 \cdot 98$ & 128 & $<16$ \\
\hline 164 & 28 & M & Normal & Endemic area & $1 \cdot 04$ & $0 \cdot 81$ & $2 \cdot 53$ & 64 & $<16$ \\
\hline 175 & 30 & $\mathrm{~F}$ & Normal & Endemic area & $0 \cdot 5$ & $0 \cdot 55$ & $1 \cdot 78$ & 64 & $<16$ \\
\hline 174 & 34 & $\mathrm{~F}$ & Normal & Endemic area & $1 \cdot 06$ & $0 \cdot 84$ & $2 \cdot 88$ & 256 & $<16$ \\
\hline 165 & 35 & $\mathrm{~F}$ & Normal & Endemic area & $1 \cdot 56$ & $0 \cdot 56$ & $1 \cdot 95$ & 64 & $<16$ \\
\hline 168 & 36 & M & Normal & Endemic area & $1 \cdot 53$ & $0 \cdot 89$ & $2 \cdot 91$ & 32 & $<16$ \\
\hline 169 & 40 & $\mathrm{~F}$ & Normal & Endemic area & $1 \cdot 23$ & $0 \cdot 81$ & $2 \cdot 45$ & 64 & $<16$ \\
\hline 186 & 42 & M & LAFB & Palpitations & $0 \cdot 98$ & $0 \cdot 76$ & $1 \cdot 98$ & 64 & $<16$ \\
\hline 176 & 45 & $\mathrm{~F}$ & Normal & Endemic area & $0 \cdot 71$ & $0 \cdot 66$ & 2 & 128 & $<16$ \\
\hline 177 & 50 & $\mathrm{~F}$ & Normal & Endemic area & $1 \cdot 41$ & $1 \cdot 07$ & $2 \cdot 89$ & 128 & $<16$ \\
\hline 190 & 51 & $\mathrm{~F}$ & Normal & Endemic area & $1 \cdot 34$ & $0 \cdot 9$ & $2 \cdot 79$ & 128 & $<16$ \\
\hline 178 & 55 & M & $\mathrm{RBBB}$ & Endemic area & $1 \cdot 32$ & $0 \cdot 89$ & $2 \cdot 23$ & 64 & $<16$ \\
\hline 183 & 55 & M & RBBB, LAFB & Endemic area & $2 \cdot 04$ & $0 \cdot 8$ & $2 \cdot 77$ & 256 & $<16$ \\
\hline 185 & 55 & M & $\mathrm{RBBB}$ & $\mathrm{CHF}$ & $0 \cdot 92$ & $0 \cdot 8$ & $2 \cdot 61$ & 64 & $<16$ \\
\hline 189 & 55 & M & Normal & $\mathrm{CHF}$ & $1 \cdot 42$ & $0 \cdot 81$ & $2 \cdot 53$ & 64 & $<16$ \\
\hline 188 & 57 & $\mathrm{~F}$ & LAFB & $\mathrm{CHF}$ & $0 \cdot 82$ & $0 \cdot 77$ & $2 \cdot 13$ & 64 & $<16$ \\
\hline 193 & 58 & M & $\mathrm{RBBB}$ & $\mathrm{CHF}$ & $0 \cdot 96$ & $0 \cdot 76$ & $1 \cdot 94$ & 64 & 16 \\
\hline 184 & 59 & $\mathrm{~F}$ & $\mathrm{RBBB}$ & CHF & $0 \cdot 66$ & $0 \cdot 64$ & $2 \cdot 39$ & 32 & $<16$ \\
\hline 166 & 63 & $\mathrm{~F}$ & Ventricular tachycardia & Syncope & $1 \cdot 54$ & $0 \cdot 77$ & $2 \cdot 96$ & 128 & $<16$ \\
\hline
\end{tabular}

${ }^{*} \mathrm{AV}$, atrioventricular; CHF, congestive heart failure; LAFB, left anterior fascicular block; RBBB, right bundle branch block.

$\dagger$ IFA results are shown as reciprocal titres.

Surface ECG revealed right bundle branch block in eight patients and left anterior fascicular block was identified in two patients, one of whom also had a right bundle branch block. Findings of ventricular tachycardia, third degree atrioventricular block, bigeminy and atrial fibrillation were all identified in one patient. Fifteen patients had normal ECGs.

Among patients aged 49 years and younger, $14 \%(2 / 14)$ had abnormal ECG findings or clinical symptoms of Chagas' disease, while $81 \%(13 / 16)$ of patients aged 50 years and older had abnormal ECG findings and $75 \%(12 / 16)$ showed clinical symptoms of Chagas' disease. Eighty-eight per cent (14/16) of asymptomatic patients had normal ECG findings (Table 1).

\section{Clinical sensitivity of the IgG assays}

The three commercial ELISA test systems each reported all 30 samples of group I as positive, resulting in a calculated sensitivity of $100.0 \%$ for each assay. The MarDx IgG IFA reported 28/30 samples as positive, resulting in a calculated sensitivity of $93 \cdot 3 \%$ (Table 2 ). 
Table 2. Agreement, sensitivity and specificity of the commercial $T$. cruzi lgG antibody assays using the manufacturers' recommended cut-off values for each assay

\begin{tabular}{|lccc|}
\hline Test & Agreement (\%) & Sensitivity (\%) & Specificity (\%) \\
\hline CeLLabs Chagas ELISA & $100 \cdot 0$ & $100 \cdot 0(91-100)$ & $100 \cdot 0(97-100)$ \\
Hemagen Chagas ELISA & $100 \cdot 0$ & $100 \cdot 0(91-100)$ & $100 \cdot 0(97-100)$ \\
IVD Research Chagas ELISA & $94 \cdot 6$ & $100 \cdot 0(88-100)$ & $93 \cdot 0(90-93)$ \\
MarDx Chagas IgG IFA & $97 \cdot 7$ & $93 \cdot 3(84-96)$ & $99 \cdot 0(96-100)$ \\
\hline
\end{tabular}

${ }^{\star}$ Confidence interval of $95 \%$.

\section{Clinical sensitivity of the IgM IFA}

True clinical sensitivity for the MarDx IgM IFA could not be determined with the samples used in this study. One sample of group I was IgM IFA positive at a screening dilution of $1: 16$, but this was without correlation to onset of symptoms or against any other assay able to detect IgM-specific antibodies against $T$. cruzi.

\section{Clinical specificity}

Clinical specificity was determined using normal sera from individuals in a non-endemic area. The determination of specificity did not include patients with infections or immunological abnormalities that might affect the true clinical specificity. Both the CeLLabs and the Hemagen ELISAs reported all 100 samples of group IV as negative, resulting in a specificity of $100 \cdot 0 \%$. The IVD Research ELISA reported 7 samples as positive and 93 samples as negative, resulting in a specificity of $93.0 \%$. The MarDx IgG IFA reported 1 sample as positive and 99 samples as negative, resulting in a calculated specificity of $99.0 \%$ (Table 2). Although the MarDx IgM IFA had a calculated specificity of $100 \cdot 0 \%$ using normal sera, the true clinical sensitivity for the MarDx IgM IFA could not be determined with the samples used in this study.

\section{Cross-reactivity studies}

The CeLLabs ELISA showed the least cross-reactivity with sera positive for antibodies directed against other parasites, infectious agents or autoantigens. Two samples previously found to be positive for antibodies against Leishmania were reported as positive by the CeLLabs T. cruzi ELISA, at just above the positive $A_{450}$ cutoff of $0 \cdot 30\left(A_{450} 0 \cdot 36\right.$ and $\left.0 \cdot 31\right)$. Both Leishmania-serology positive samples and three of the ten SLE-serology positive samples had positive results by the Hemagen ELISA. One of the two Leishmania-serology positive, one of the ten Toxoplasma-serology positive and one of the ten SLE-serology positive samples had positive results with the IVD Research ELISA. The one sample positive for Leishmania antibodies that was reported as positive by the IVD Research EIA was just above the positive $A_{450}$ cutoff of $0 \cdot 20\left(A_{450} 0 \cdot 21\right)$. The MarDx IgG IFA showed the most cross-reactivity with other antibody-positive samples. One malaria, one Schistosoma, two Leishmania, two Toxoplasma, one rheumatoid factor, one SLE- and one DNase

Table 3. Total number of discrepant results from samples in groups II and III

No cross-reactivity was observed with any of the test systems from the following: Cysticercosis $(n=7)$, Strongyloides $(n=3)$, Brucella $(n=3)$, Toxocara $(n=2)$, E. histolytica $(n=3)$, Leptospira $(n=1)$, Trichinella $(n=1)$, syphilis $(n=10)$ or infectious mononucleosis $(n=10)$.

\begin{tabular}{|lcccccc|}
\hline \multirow{2}{*}{ Group and disease } & $\begin{array}{c}\text { Total no. of } \\
\text { samples }\end{array}$ & \multicolumn{5}{c|}{ Total no. (and percentage) of positive results } \\
\cline { 3 - 6 } & & $\begin{array}{c}\text { CeLLabs } \\
\text { ELISA }\end{array}$ & $\begin{array}{c}\text { Hemagen } \\
\text { ELISA }\end{array}$ & $\begin{array}{c}\text { IVD Research } \\
\text { ELISA }\end{array}$ & $\begin{array}{c}\text { MarDx } \\
\text { IgG IFA }\end{array}$ & $\begin{array}{c}\text { MarDx } \\
\text { IgM IFA }\end{array}$ \\
\hline $\begin{array}{l}\text { Group II } \\
\text { Malaria }\end{array}$ & 3 & $0(0)$ & $0(0)$ & $0(0)$ & $1(33)$ & $0(0)$ \\
$\begin{array}{l}\text { Schistosoma } \\
\text { Leishmania }\end{array}$ & 5 & $0(0)$ & $0(0)$ & $0(0)$ & $1(20)$ & $0(0)$ \\
$\begin{array}{l}\text { Toxoplasma } \\
\text { Group III }\end{array}$ & 2 & $2(100)$ & $2(100)$ & $1(50)$ & $2(100)$ & $0(0)$ \\
$\begin{array}{l}\text { Rheumatoid arthritis } \\
\text { Systemic lupus }\end{array}$ & 10 & $0(0)$ & $0(0)$ & $1(10)$ & $2(20)$ & $0(0)$ \\
$\quad$ erythematosus & 10 & $0(0)$ & $0(0)$ & $0(0)$ & $1(10)$ & $0(0)$ \\
Rheumatic fever & 10 & $0(0)$ & $3(30)$ & $1(10)$ & $1(10)$ & $0(0)$ \\
& 10 & $0(0)$ & $0(0)$ & $0(0)$ & $1(10)$ & $0(0)$ \\
\hline
\end{tabular}


B- (rheumatic fever) serology positive sample had positive results in the MarDx IgG IFA (Table 3). No cross-reactivity was observed with any of the test systems from the following infection categories: Cysticercosis $(n=7)$, Strongyloides $(n=3)$, Brucella $(\mathrm{n}=3)$, Toxocara $(n=2)$, E. histolytica $(n=3)$, Leptospira $(n=1)$, Trichinella $(n=1)$, syphilis $(n=10)$ or infectious mononucleosis $(n=10)$.

\section{Reproducibility studies}

Intra- and inter-assay precision studies were performed for the CeLLabs, Hemagen and IVD Research ELISAs. For the three ELISAs, three samples were used for the precision studies, a negative, a low positive and a medium positive sample. All of the positive samples remained positive on retesting. For these samples, the CV (coefficient of variation) ranged from $2 \cdot 4$ to $13 \cdot 5 \%$. The IVD Research assay showed the highest $\mathrm{CV}$. The inter-assay $\mathrm{CV}$ was $7 \cdot 2 \%$ for the low positives (mean $1.5 \pm 0 \cdot 11 \mathrm{SD}$ ), and $13.5 \%$ (mean $2 \cdot 0 \pm$ $0 \cdot 27 \mathrm{SD}$ ) for the medium positives. The negative samples remained negative (mean $0 \cdot 03 \pm 0 \cdot 04 \mathrm{SD}$ ). For IgG and IgM IFA, the sample results were within one well of the mean titre for each replicate.

\section{Comparison of the ELISA test systems}

Quantitative ELISA results $\left(A_{450}\right.$ readings or calculated index values) were subdivided into groups I-IV as defined previously (Fig. 1). The IVD Research ELISA showed the greatest range of $A_{450}$ results, from $0 \cdot 0$ to $3 \cdot 16$. The CeLLabs ELISA showed the second greatest $A_{450}$ range, from $0 \cdot 0$ to
2.04. The Hemagen ELISA showed the narrowest range of results, with index values from 0.0 to $1 \cdot 07$. When the manufacturers' recommended cut-off values were employed, the CeLLabs assay showed the best overall results. The results for group I samples were separated from the results of groups II to IV samples, with the exception of the two Leishmania samples that were above the positive result cut-off.

Laboratory assays are essential for the accurate diagnosis of Chagas' disease. During acute infections when the level of parasitaemia is highest, direct detection through buffy coat examination (Kirchhoff, 1996; Tanowitz et al., 1992), PCR assays (Kirchhoff et al., 1996; Tanowitz et al., 1992) or xenodiagnosis (Leiby et al., 2000) can be accomplished. Additionally, IgM antibody detection may be useful (Kirchhoff, 1996; Kirchhoff et al., 1996). During the indeterminate or chronic stage of T. cruzi infection, the level of actual parasites is low, hence detection rests solely on the presence of IgG antibody against T. cruzi (Tanowitz et al., 1992). Although direct identification of T. cruzi through blood smear analysis, PCR assays or xenodiagnosis is highly specific, these methods are often laborious and unsuitable for use in a reference laboratory. Antibody testing is well suited for a reference laboratory setting, and current ELISAs offer high performance characteristics in T. cruzi antibody detection. Both the CeLLabs and the Hemagen Chagas ELISAs had agreement, sensitivity and specificity of $100 \cdot 0 \%$. However, Leishmania-positive samples, as well as SLE-positive samples, showed cross-reactivity in the Hemagen and IVD

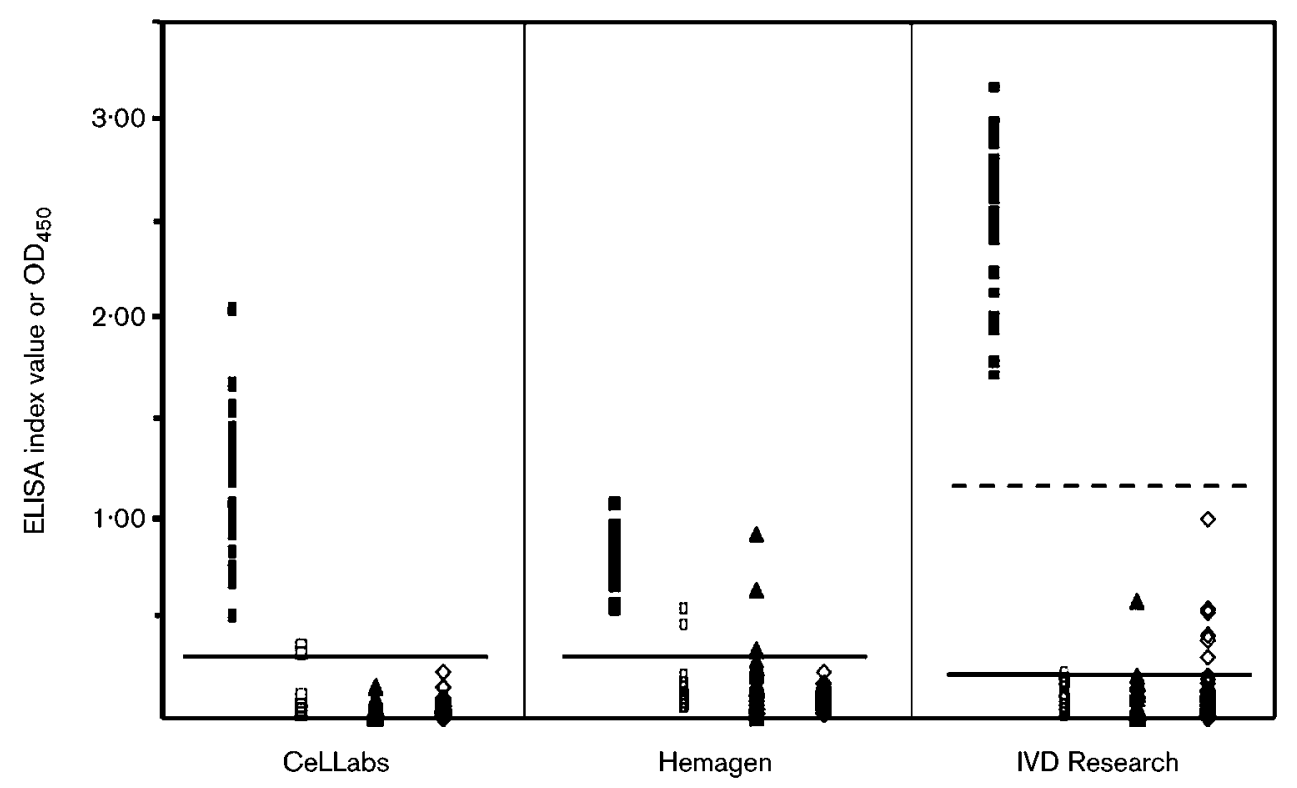

Fig. 1. Distribution of results obtained with the CeLLabs, Hemagen and IVD Research ELISA test systems for the serum samples from group I (Chagas-serology positive), group II (parasite-serology positive), group III (infectious disease or autoimmune-serology positive) and group IV (normal negative donors). A solid horizontal line identifies the manufacturer's recommended cut-off for each assay. A dashed horizontal line identifies our revised cut-off for the IVD Research ELISA. $\mathbf{}$, Group I; $\square$, group II; $\boldsymbol{\Delta}$, group III; $\diamond$, group IV. 
Research assays (Table 3). Skeiky et al. (1992) pointed out similarities between proteins produced by patients infected with T. cruzi and SLE, and cross-reactivity between T. cruzi and Leishmania samples in antibody assays is well documented (Chiller et al., 1990; Kirchhoff, 1996; Reiche et al., 1998; Solana et al., 1995).

Due to the low specificity in EIAs, Latin American laboratories are instructed to test samples from chagasic patients using at least two methodologies (Mocayo, 1992; Schmunis, 1991). By coupling several assays with acceptable sensitivities and specificities, it is hoped that any antibody-positive and thus $T$. cruzi-positive patients will be accurately identified, and thus precluded from donating blood. IFA is commonly used in Latin American laboratories as one of the two tests for Chagas' disease serological diagnosis (Oelemann et al., 1998). However, it is generally less sensitive and more subjective than ELISA tests. A variety of fluorescence patterns from cross-reacting antibodies make IFA interpretation very challenging and can result in false-positive results.

The IgM IFA is the only commercial assay that is available for detection of IgM-specific T. cruzi antibodies. A positive IgM IFA result is indicative of acute infection (Camargo \& Amato Neto, 1974; Reyes et al., 1990; Umezawa et al., 1996a, b, c), and hence an IgM assay could be an important diagnostic tool. But it is suspected that the antigens used in most IgG assays may not be specific enough for IgM assays, which would lower the overall sensitivity (Umezawa et al., 1996c). A negative result by the MarDx IgM IFA could mean the IgM antibody response has faded over time, the IFA is not sensitive enough to detect the low amount of IgM that may be present, or the assay is not manufactured using the best combination of antigens. As discussed by Umezawa et al. (1996c), purified surface trypomastigote antigens used in ELISAs may be both sensitive and specific enough to diagnose acute T. cruzi infections. Additionally, clinical diagnosis of acute infection is uncommon due to the asymptomatic course of the disease, and by the time chronic infection is evident, IgM antibody has been absent for years or decades. An exception has been in cases of transfusionrelated infection. When immunosuppressed patients receive T. cruzi-contaminated blood products, a fulminant illness usually results (Kirchhoff, 1996) and symptoms are easily recognized. Although IgM testing has its place in laboratory diagnosis of acute T. cruzi infection and is useful for the diagnosis of congenital infection, a better overall approach is in testing paired acute and convalescent samples for IgG antibodies. The majority of $T$. cruzi antibody/antigen relationship research has been performed using IgG antibodies, thus IgG assays have been extensively developed and generally perform better. Some patients remain seronegative all their lives (Camargo, 1992), however, so for diagnosis, immunological assays should always be coupled with ECG data and clinical symptoms. Further research into IgM assays should be pursued so that the best possible product can be made available.
Confirmation of a positive serology result for T. cruzi is still difficult. Xenodiagnosis and haemoculture are highly impractical (Leiby et al., 2000). The radioimmunoprecipitation assay appears to be very promising and specific (Leiby et al., 2000), as does Western blotting (Reiche et al., 1998; Solana et al., 1995; Umezawa et al., 1996c), although these assays are not available commercially. PCR is more sensitive than xenodiagnosis (Avila et al., 1993) and microscopic examination (Kirchhoff, 1996). However, a negative PCR result does not necessarily exclude infection. Because the parasitaemia is very low in chronic infection, it is unlikely that PCR will be useful in the chronic stage.

Unlike the Hemagen assay, which is FDA approved for diagnostic purposes only (not for blood donor screening), the IVD IgG ELISA and the CeLLabs IgG ELISA are labelled as analyte-specific reagents (ASR). The clinical reference ranges of ASR assays may be adjusted after assessment of the performance characteristics according to the FDA (Jaros, 1992; Litwin, 2000; Paxton, 1998). Therefore, if cut-off values are adjusted based on the results collected for the samples in groups I to IV, the IVD Research ELISA outperformed the Hemagen and CeLLabs ELISAs. The IVD Research ELISA showed the greatest separation between positive and negative results; samples from group I had results that ranged from $A_{450} 1 \cdot 71$ to $3 \cdot 16$, while samples from groups II to IV had results that ranged from $A_{450} 0 \cdot 0$ to $1 \cdot 0$. The Hemagen ELISA showed the highest values for cross-reactivity. If an index value of greater than 0.50 was obtained in the Hemagen ELISA, it would not be possible to confidently report the result as positive for T. cruzi antibody versus a cross-reactive antibody. If an $A_{450}$ of greater than $1 \cdot 20$ was obtained in the IVD Research ELISA, however, the sample had a high likelihood of being a true positive T. cruzi result (Fig. 1). Therefore, if the cut-off value for this ELISA is changed to $1 \cdot 20$, the agreement and specificity of the assay would change from $94 \cdot 6 \%$ and $93.0 \%$, respectively, to $100 \%$ for both.

It is possible that the manufacturer's cut-off value of $0 \cdot 20$ for the IVD IgG ELISA may have been based on studies that included weakly positive serum samples from patients with Chagas' disease, whereas our dataset may have included more strongly positive sera. Taken together, we think it might be useful to include an equivocal $A_{450}$ range of $0 \cdot 2-$ $1 \cdot 19$. Further studies with a higher number of previously classified sera (high, medium and low antibody titre) will be needed to refine this range. An equivocal result would indicate to the clinician the possibility of low level Chagas' antibodies, cross-reactivity with another parasite or a nonspecific interfering substance.

All of the assays presently available in the United States have an adequate ability to discriminate positive and negative tests. The IVD Research ELISA showed the best separation between positive and negative results, and the least crossreactivity, using an adjusted cut-off value. Based on the clear separation between positive and negative serum samples, an equivocal range encompassing results between the 
recommended manufacturer's cut-off and the adjusted cutoff suggested would more likely detect antibodies that are cross-reactive with T. cruzi, such as seen in leishmaniasis, rather than true positives. Overall the CeLLabs assay had the best performance of the three kits when following the manufacturer's instructions and established cut-offs.

\section{ACKNOWLEDGEMENTS}

We would like to thank the staff at the Chagas clinic and laboratory service at the Oswaldo Cruz University hospital in Recife, Brazil for their gracious assistance. This work was supported by the ARUP Institute for Clinical and Experimental Pathology and by a Public Health Service grant AI40067 from the National Institute of Allergy and Infectious Diseases to C. M. L.

\section{REFERENCES}

Avila, H. A., Pereira, J. B., Thiemann, O., De Paiva, E., DeGrave, W., Morel, C. M. \& Simpson, L. (1993). Detection of Trypanosoma cruzi in blood specimens of chronic chagasic patients by polymerase chain reaction amplification of kinetoplast minicircle DNA: comparison with serology and xenodiagnosis. J Clin Microbiol 31, 2421-2426.

Camargo, M. E. (1992). Serological diagnosis - an appraisal of Chagas' disease serodiagnosis. In Chagas' Disease (American Trypanosomiasis): its Impact on Transfusion and Clinical Medicine, pp. 165178. Edited by A. Rassi. Sao Paulo, Brazil: Cartgraf Editora Ltd.

Camargo, M. E. \& Amato Neto, V. (1974). Anti-Trypanosoma cruzi IgM antibodies as serological evidence of recent infection. Rev Inst Med Trop Sao Paulo 16, 200-202.

Centers for Disease Control (CDC) (2002). Chagas disease after organ transplantation - United States, 2001. Morb Mortal Wkly Rep 51, 210-212.

Chiller, T. M., Samudio, M. A. \& Zoulek, G. (1990). IgG antibody reactivity with Trypanosoma cruzi and Leishmania antigens in sera of patients with Chagas' disease and leishmaniasis. Am J Trop Med Hyg 43, 650-656.

Ferreira, A. W., Belem, Z. R., Lemos, E. A., Reed, S. G. \& CamposNeto, A. (2001). Enzyme-linked immunosorbent assay for serological diagnosis of Chagas' disease employing a Trypanosoma cruzi recombinant antigen that consists of four different peptides. J Clin Microbiol 39, 4390-4395.

Freilij, H. \& Altcheh, J. (1995). Congenital Chagas' disease: diagnostic and clinical aspects. Clin Infect Dis 21, 551-555.

Jaros, M. (1992). Guidelines for evaluating and introducing new clinical tests. Clin Immunol Newsl 12, 33-37.

Kerndet, P. H. A. W., Kirchhoff, L. V., Steurer, F. J., Waterman, S. Nelson, J., Gelert, G. \& Shulman, I. (1991). Prevalence of antibody to Trypanosma cruzi among blood donors in Los Angeles, California. Transfusion 31, 814-818.

Kirchhoff, L. V. (1993). American trypanosomiasis (Chagas' disease) - a tropical disease now in the United States. N Engl J Med 329, 639-644.

Kirchhoff, L. V. (1996). American trypanosomiasis (Chagas' disease). Gastroenterol Clin North Am 25, 517-533.

Kirchhoff, L. V., Votava, J. R., Ochs, D. E. \& Moser, D. R. (1996). Comparison of PCR and microscopic methods for detecting Trypanosoma cruzi. J Clin Microbiol 34, 1171-1175.

Krauss, H., Weber, A., Appel, M., Enders, B., Isenberg, H. D., Schiefer, H. G., Slenczka, W., von Graevenitz, A. \& Zahner, H. (editors) (2003). Parasitic zoonoses. In Zoonoses: Infectious Diseases Transmissible from Animals to Humans, 3rd edn, pp. 273-277. Washington, DC: ASM Press.

Leiby, D. A., Read, E. J., Lenes, B. A., Yund, A. J., Stumpf, R. J., Kirchhoff, L. V. \& Dodd, R. Y. (1997). Seroepidemiology of Trypanosoma cruzi, etiologic agent of Chagas' disease, in US blood donors. J Infect Dis 176, 1047-1052.

Leiby, D. A., Wendel, S., Takaoka, D. T., Fachini, R. M., Oliveira, L. C. \& Tibbals, M. A. (2000). Serologic testing for Trypanosoma cruzi: comparison of radioimmunoprecipitation assay with commercially available indirect immunofluorescence assay, indirect hemagglutination assay, and enzyme-linked immunosorbent assay kits. J Clin Microbiol 38, 639-642.

Litwin, C. M. (2000). Guidelines for verification and validation of immunology tests. Clin Microbiol Newsl. 22, 173-176.

Martins, T. B., Jaskowski, T. D., Mouritsen, C. L. \& Hill, H. R. (1995). An evaluation of the effectiveness of three immunoglobulin G (IgG) removal procedures for routine IgM serological testing. Clin Diagn Lab Immunol 2, 98-103.

McCarthy, M. (2003). American Red Cross to screen blood for Chagas' disease. Lancet 362, 1988.

Mendes, R. P., Hoshino-Shimizu, S., Moura da Silva, A. M., Mota, I., Heredia, R. A., Luquetti, A. O. \& Leser, P. G. (1997). Serological diagnosis of Chagas' disease: a potential confirmatory assay using preserved protein antigens of Trypanosoma cruzi. J Clin Microbiol 35, 1829-1834.

Mocayo, A. (1992). Chagas' disease. Epidemiology and prospects for interruption of transmission in the Americas. World Health Stat Q 45, 276-279.

Oelemann, W. M., Teixeira, M. D., Verissimo Da Costa, G. C., BorgesPereira, J., De Castro, J. A., Coura, J. R. \& Peralta, J. M. (1998). Evaluation of three commercial enzyme-linked immunosorbent assays for diagnosis of Chagas' disease. J Clin Microbiol 36, 2423-2427.

Paxton, H. (1998). Method validation and verification for highcomplexity and inhouse (home brew) diagnostic immunology tests. In Clinical Diagnostic Immunology: Protocols in Quality Assurance and Standardization, pp. 71-77. Edited by J. L. Sever. Malden, MA: Blackwell Science, Inc.

Reiche, E. M., Cavazzana, M. Jr, Okamura, H., Tagata, E. C., Jankevicius, S. I. \& Jankevicius, J. V. (1998). Evaluation of the western blot in the confirmatory serologic diagnosis of Chagas' disease. Am J Trop Med Hyg 59, 750-756.

Reyes, M. B., Lorca, M., Munoz, P. \& Frasch, A. C. (1990). Fetal IgG specificities against Trypanosoma cruzi antigens in infected newborns. Proc Natl Acad Sci U S A 87, 2846-2850.

Schechter, M., Voller, A., Marinkelle, C. J., Flint, J. E., Guhl, F. \& Miles, M. A. (1983). Purified Trypanosoma cruzi specific glycoprotein for discriminative serological diagnosis of South American trypanosomiasis (Chagas' Disease). Lancet 2, 939-941.

Schmunis, G. A. (1991). Trypanosoma cruzi, the etiologic agent of Chagas' disease: status in the blood supply in endemic and nonendemic countries. Transfusion 31, 547-557.

Shikanai-Yasuda, M. A., Marcondes, C. B., Guedes, L. A. \& 10 other authors (1991). Possible oral transmission of acute Chagas' disease in Brazil. Rev Inst Med Trop Sao Paulo 33, 351-357.

Skeiky, Y. A. W., Benson, D. R., Parsons, M., Elkon, K. B. \& Reed, S. G. (1992). Cloning and expression of Trypanosoma cruzi ribosomal protein $\mathrm{P} 0$ and epitope analysis of anti-P0 autoantibodies in Chagas' disease patients. J Exp Med 176, 201-211.

Solana, M. E., Katzin, A. M., Umezawa, E. S. \& Miatello, C. S. (1995). High specificity of Trypanosoma cruzi epimastigote ribonucleoprotein as antigen in serodiagnosis of Chagas' disease. J Clin Microbiol 33, 1456-1460. 
Tanowitz, H. B., Kirchhoff, L. V., Simon, D., Morris, S. A., Weiss, L. M. \& Wittner, M. (1992). Chagas' disease. Clin Microbiol Rev 5, 400-419.

Umezawa, E. S., Shikanai-Yasuda, M. A. \& Stolf, A. M. (1996a). Changes in isotype composition and antigen recognition of antiTrypanosoma cruzi antibodies from acute to chronic Chagas disease. J Clin Lab Anal 10, 407-413.

Umezawa, E. S., Shikanai-Yasuda, M. A., Gruber, A., PereiraChioccola, V. L. \& Zingales, B. (1996b). Trypanosoma cruzi defined antigens in the serological evaluation of an outbreak of acute Chagas disease in Brazil (Catole do Rocha, Paraiba). Mem Inst Oswaldo Cruz 91, 87-93.

Umezawa, E. S., Nascimento, M. S., Kesper, N. Jr, Coura, J. R., Borges-Pereira, J., Junqueira, A. C. \& Camargo, M. E. (1996c). Immunoblot assay using excreted-secreted antigens of Trypanosoma cruzi in serodiagnosis of congenital, acute, and chronic Chagas' disease. J Clin Microbiol 34, 2143-2147.

World Health Organization (WHO) (1991). Control of Chagas disease: report of a WHO expert committee. Geneva, Switzerland: World Health Organization. 